Original Research Paper

\title{
Dynamical System of Flat Electroencephalography
}

\author{
${ }^{1}$ Tan Lit Ken, ${ }^{2}$ Tahir bin Ahmad, ${ }^{3}$ Mohd Sham bin Mohamad and ${ }^{4}$ Su Kong Ngien \\ ${ }^{I}$ Department of Mechanical Precision Engineering, \\ Frontier Materials Research Alliance, Malaysia-Japan International Institute of Technology, \\ Universiti Teknologi Malaysia, UTM KL, Jalan Sultan Yahya Petra, 54100 Kuala Lumpur, Malaysia \\ ${ }^{2}$ Department of Mathematical Science and Ibnusina Institute, \\ Universiti Teknologi Malaysia, 81310 Skudai, Johor Darul Takzim, Malaysia \\ ${ }^{3}$ Science Programme, Faculty of Industrial Sciences and Technology, \\ ${ }^{4}$ Faculty of Civil Engineering and Earth Resources, \\ Universiti Malaysia Pahang, Lebuhraya Tun Razak, 6300 Gambang Kuantan, Pahang Darul Makmur, Malaysia
}

Article history

Received: 16-04-2015

Revised: $30-07-2015$

Accepted: 31-07-2015

Corresponding Author:

Tan Lit Ken

Department of Mechanical

Precision Engineering, Frontier

Materials Research Alliance,

Malaysia-Japan International

Institute of Technology, UTM

KL, Jalan Sultan Yahya Petra,

54100 Kuala Lumpur, Malaysia

Email: tlken@utm.my

\begin{abstract}
Flat EEG is a way of viewing electroencephalography signals on the real plane. The wealth of information contained within makes it a great platform to study epileptic seizure. In this study, the time embedment nature of Flat EEG is exploited to establish a dynamic Flat EEG model. Basically, a geometrical representation which displays the important information visually for Flat EEG at any time will firstly be formulated, followed by the representation of a Flat EEG signal as a trajectory in state space. Based on these, the modeling of Flat EEG as a dynamical system using the notion of flow is presented. This dynamic model would serve as the groundwork for the indisputably essential dynamic justification of Flat EEG in future research.
\end{abstract}

Keywords: Flat EEG, Dynamic, Trajectory, State Space, Modelling

\section{Introduction}

Epilepsy is one of the most common brain disorders in the world. This disorder can happen to anyone in the world at any age regardless of gender. The Commission on Epidemiology and Prognosis and International League Against Epilepsy 1993 defined epilepsy as "occurrence of at least two unprovoked seizure" (Panayiotopoulos, 2010), that is, it usually occurs more than once and is not prompted. This brain disorder is not contagious i.e., cannot be transmitted or spread from one person to another. Statistically, the number of people with epilepsy in the world is at least 50 million in total which is roughly $1.5 \%$ of the world population (Jahnecke et al., 2007).

Epilepsy is not a form of mental illness and it generally does not affect the ability to think and learn. In fact, most people with epilepsy have normal mental intelligence, with some having superior cognitive abilities (Wyllie et al., 2006). Nevertheless, epilepsy can limit one's independence, freedom and quality of life. For example, a person with epilepsy has limited job options, have trouble in getting a driving license, faces social rejection, discrimination or prejudice due to other people's fears and misconceptions about their condition. Epilepsy is dangerous as it can lead to fatality especially when a seizure continues for a long period of time and causes brain damage. Such a situation is known as status epilepticus.

Two of the methods for controlling or stopping seizures are taking anticonvulsant medications and undergoing surgery. The former method is challenging in terms of finding the exact combination of medications and dosage. Besides, this method is not promising because some seizures can still fail to be controlled in spite of the best available medication. Moreover, anticonvulsant medications carry undesired side effects (Sharon et al., 2004; Stefan and Feuerstein, 2007). The effects are based on the type of anticonvulsant medications used which in turn are based on the type of epilepsy seizure. Frequently, it adversely affects the cognitive ability of patients (Bennett, 1992). Consequently, some patients choose to undergo epilepsy surgery. As a matter of fact, neurologists often suggest surgery as the best solution. The target of this method is to remove problematic epileptogenic tissue while sparing essential brain areas to avoid neurologic deficits. Therefore, knowing the exact location of problematic cells i.e., epileptic foci is crucial.

Electroencephalography is the process of obtaining electrical potentials produced by neurons inside the brain. This harmless electrophysiological process which uses a machine called electroencephalograph (Fig. 1) is usually performed by a certified technologist. 


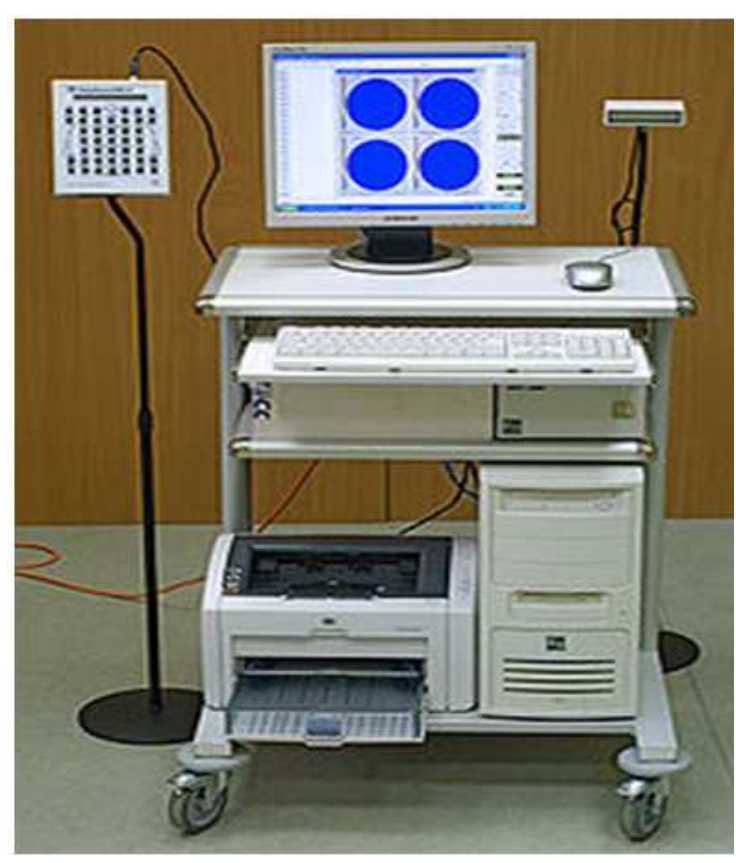

Fig. 1. EEG signal (www.wikipedia.com)

With this instrument, the summed electrical activity which results from the ionic current flows between neurons during the transmission of information underneath the electrodes can be recorded noninvasively from the scalp. Usually, recordings only last for 20 to 40 min (including preparation time). Electroencephalography is important in neurosurgery as it is able to detect abnormal patterns of electrical activity inside the brain. The advantage of this technology, other than being harmless in diagnosing epileptic seizure is that it is also one of the most economic methods (Kiymik et al., 2005).

\section{Literature Review}

Various research using different concepts and techniques to identify epileptic foci has been established in the interest of creating better life for epileptic patients. For example, via multimodality approach (Desco et al., 2001), by using large-area magnetometer and functional brain anatomy (Tiihonen et al., 2004), examining correlations among electrodes captured by linear, nonlinear and multi linear data analysis technique (Evim et al., 2006), 3-D source localization of epileptic foci by integrating EEG and MRI data (Natasa et al., 2003) and even approaches that are based on statistical tools such as Bayesian method (Toni et al., 2005) and maximum likelihood estimation approach by Jan et al. (2004). Each of the methods has their own advantages and weaknesses.

Fuzzy Topographic Topological Mapping (FTTM) is a fuzzy and topological based model for solving neuromagnetic inverse problem (Fig. 2). Consisting of four components i.e., Magnetic Contour Plane (MC), Base Magnetic Plane (BM), Fuzzy Magnetic Field (FM) and Topographic Magnetic Field (TM), each of these components are homeomorphic to one another (Liau, 2001). For recorded data, the model is capable of portraying current sources topographically in three dimensions space. The advantage of this method is that, it does not need prior information and it is not time consuming (Tahir et al., 2005).

To study the brain disorder more effectively, a novel method called Flat electroencephalography (Flat EEG) (Fig. 3) was invented to view EEG signals on the first component of Fuzzy Topographic Topological Mapping (FTTM). Thus, theoretically, by FTTM model, EEG signals can be portrayed in 3-dimension space. This method consists of a flattening procedure (a stereographic projection) which serves as the transformation from EEG to MC (Fauziah, 2008) (Fig. 4). The main scientific value of this method lies in its ability to preserve the orientation and magnitude of EEG signals to MC, allowing it to be compressed and analyzed. Hence, performing analysis on EEG signals would allow researchers to reveal interesting patterns contained within the signals.

Basically, Flat EEG is a platform which enables EEG signals be studied on the Cartesian plane mathematically, hence allowing the extraction of "hidden" information within EEG signals that cannot be obtained by traditional visual inspection. Furthermore, characteristics and properties obtained from this platform can be used to describe epileptic seizure since epileptic seizure and Flat EEG are topologically conjugated (Tahir and Tan, 2010) (Fig. 5).

There have been numerous researches utilizing various mathematical tools to visualize and extract "hidden" information within EEG signals via Flat EEG on a particular frame (or time) with promising outcome. For instance, implementation of Fuzzy C-Means (FCM) on Flat EEG enables one to compute the number of cluster centers along with its locations, hence made tracking brainstorm during epileptic seizure possible (Fauziah, 2008).

Apart from that, the algebraic study on Flat EEG demonstrates not only the possibility of transforming Flat EEG from one mathematical structure to another i.e., from topological to algebraic but also shows that Flat EEG can be decomposed into semigroup of upper triangular matrices under matrix multiplication and hence revealing that patterns exist in epileptic seizure process rather than chaotic (Faisal and Tahir, 2010).

Furthermore, study on the dynamic structure of Flat EEG, in particular, its structural stability from topological viewpoint, proves that Flat EEG in the presence of artifacts could still offer significant descriptions of electrical activities in the brain during seizure attack (Tan and Tahir, 2014) (Fig. 6). 


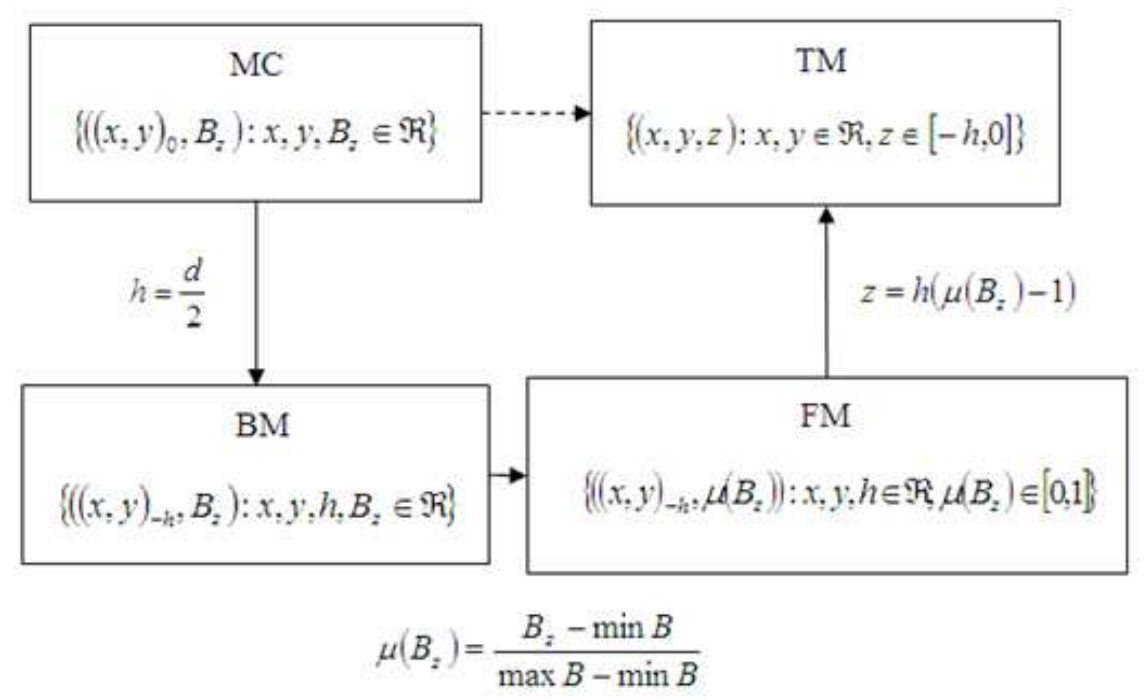

Fig. 2. FTTM

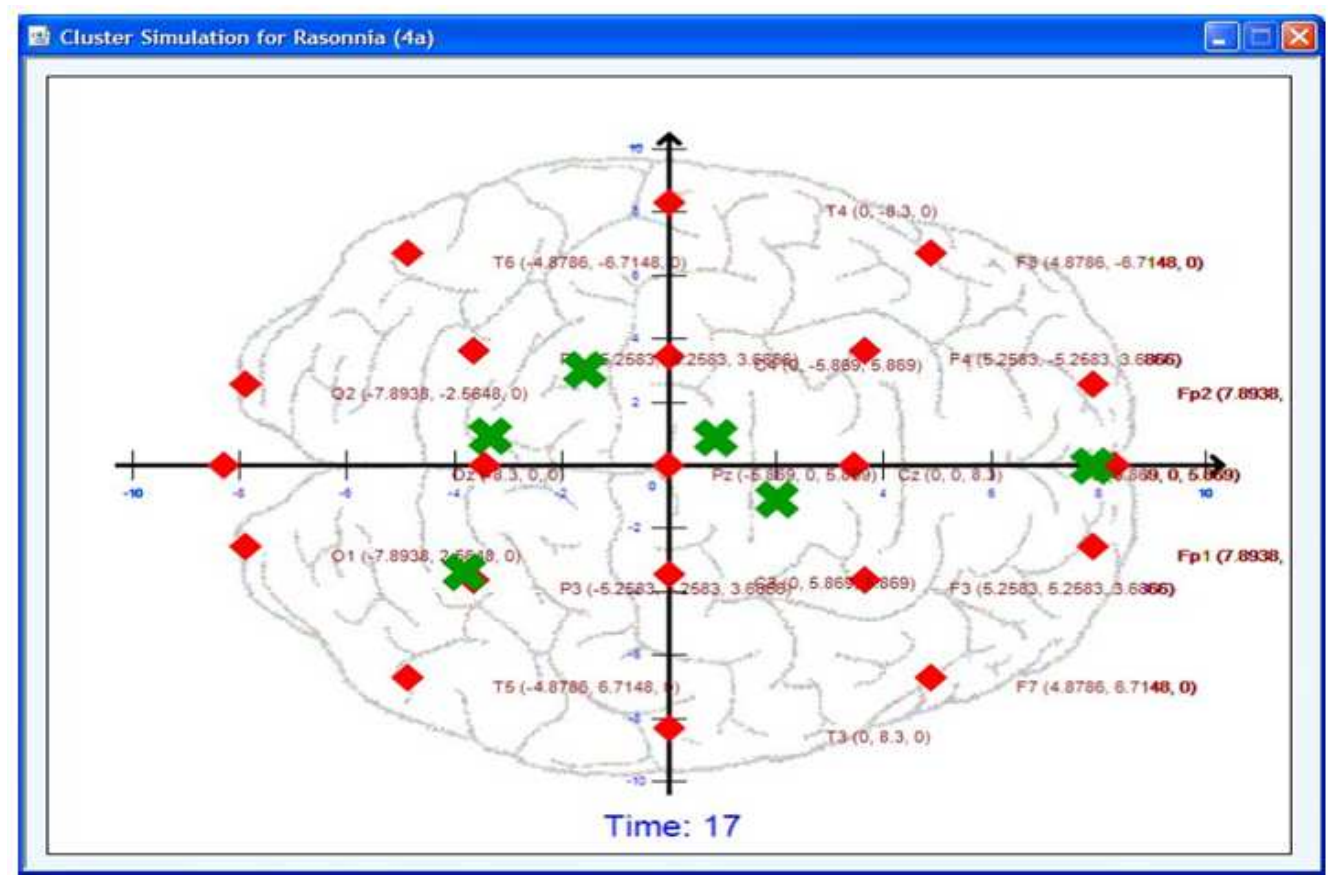

Fig. 3. A flat EEG at $t=3$

Modelling is the scientific way of studying real world phenomena to gain deeper understanding on it. It is the idealization (or simplification) of a system in reality i.e., it usually incorporates only significant characteristics. Since the importance of those characteristics is subjective, various models can be built for a phenomenon and the success of a model depends on the accuracy of its predictions (Edwards and Hamsom, 2001). Modelling allows the properties and behavior of any situation be studied economically and easily in an abstract form (Samarskii and Mikhailov, 2002).
The main goal of modelling is to construct a model that represents the reality as closely as possible. However, it is often not possible to incorporate all features due to the complexity of the phenomenon. Consequently, simplification or idealization is a natural step in modelling (Gibbons, 1995; Kalman, 1997). Indeed, a model is simply the assumptions that the modeler made. Simplifications are often executed to discard features that are not very relevant and significant, retaining only essential features of the problems. 


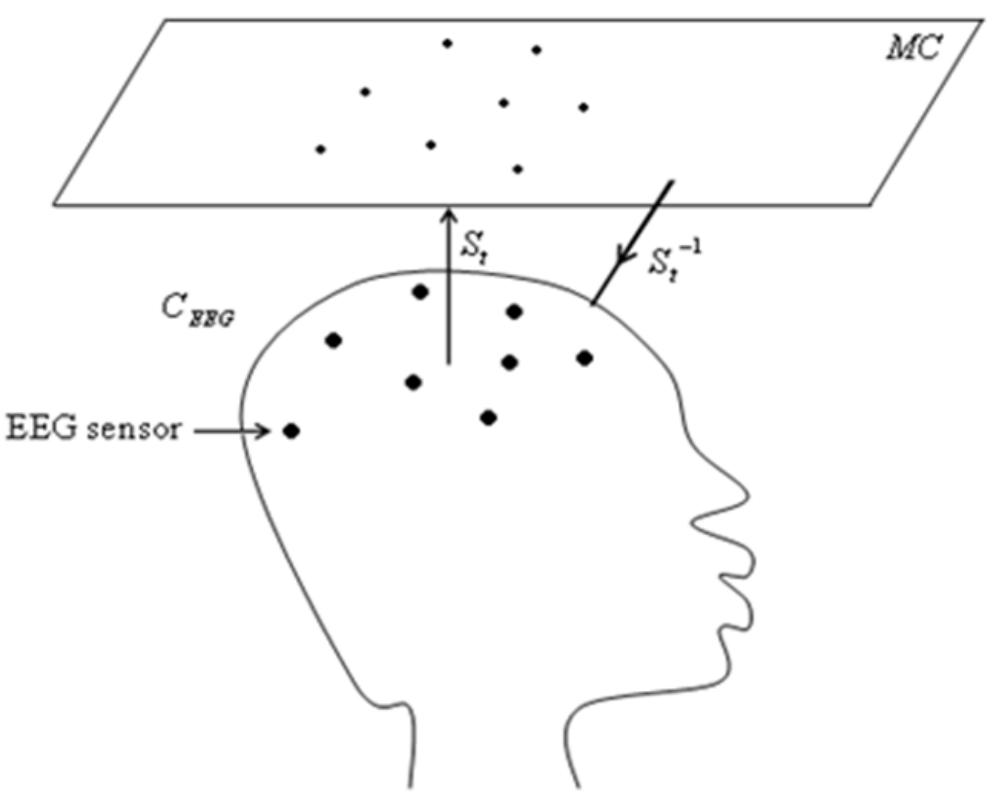

Fig. 4. Stereographic projection
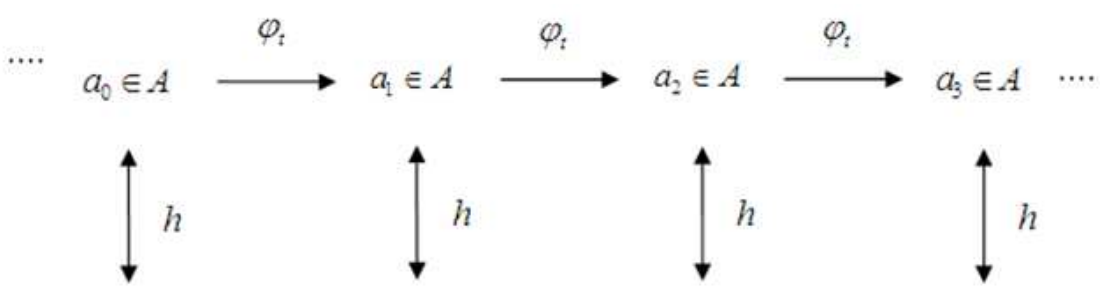

$\cdots \quad b_{0} \in B \longrightarrow{ }_{\psi_{i}}^{\longrightarrow} b_{0} \in B \longrightarrow{ }_{\psi_{i}}^{\longrightarrow} b_{0} \in B \underset{\psi_{t}}{\longrightarrow} b_{0} \in B$

Fig. 5. Pictorial representation of topological conjugacy

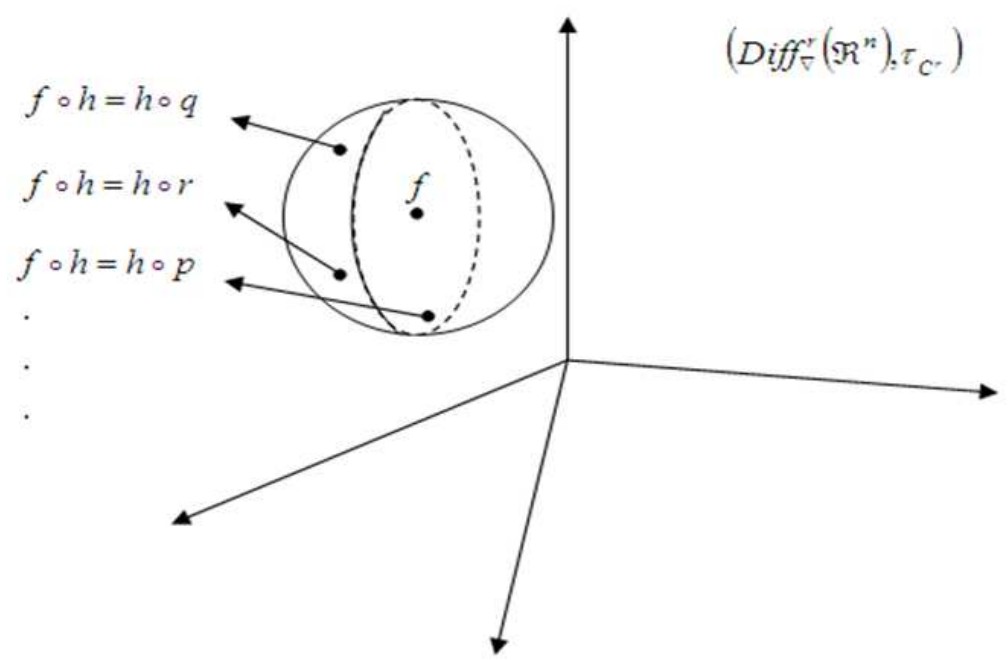

Fig. 6. A structurally stable diffeomorphism $f$ in $\left(\operatorname{Diff} f_{\nabla}^{r}\left(\Re^{n}\right), \tau_{C^{r}}\right)$ 


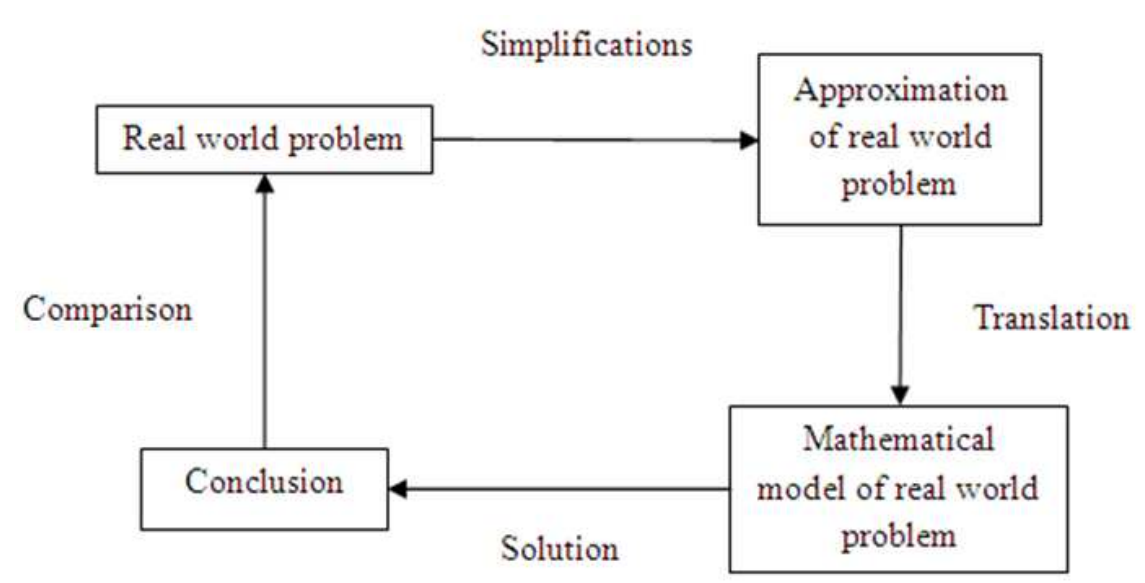

Fig. 7. Mathematical modeling

According to Jagat (1988), the justification for these simplifications can usually be found from the closeness of agreement between observation and approximated model.

Mathematical models are models constructed using mathematical theories (Edwards and Hamsom, 2001). In mathematical modelling, real world phenomenon is usually transformed into abstract mathematical world, usually in the form of functions, equations or inequalities, where plentiful of mathematic tools are available for subsequent analysis. These tools are usually developed by mathematicians who have little thought on how it will eventually be used (Burghes et al., 1996). In short, mathematical modelling utilizes mathematical tools to test ideas and make predictions about the real world (Fowkes and Mahony, 1994).

Mathematical modelling can generally be divided into four steps (Jagat, 1988) (Fig. 7) they are:

- Simplifications-Identify significant characteristics and make assumptions i.e., make connections between related variables

- Translation-Write assumptions in mathematical equations

- Solving-Manipulate and solve the problems using available tools

- Comparison-Interpret and check the results obtained to see the degree of agreement, correspondence and validity

A dynamical system is a system whose temporal evolution from some initial state is dictated by a set of rules (Eduard et al., 1999). Another way to understand this is, it consists of a set of variables that describe its state and a law that describe the evolution of the state variables with time, i.e., how the state of the system in the next moment of time depends on the input and its state in the previous moment of time (Eugene, 2007).
Flat EEG is a model and a visual entity that progress through time. By the definition of dynamical system, this shows that it is a model of a dynamical system, particularly flow. This is certainly appropriate since Flat EEG is potentially generated by differential equations (Basar et al., 1988; Fell et al., 1996; Thomasson et al., 2001; Song et al., 2004). Thus, in this study, Flat EEG will be modeled as a dynamical system. The establishment of dynamic model for Flat EEG would serve as the groundwork for dynamic topological transformation from epileptic seizure to Flat EEG i.e., to justify Flat EEG as an alternative platform to study epileptic seizure. Besides, it will also allow the structural stability of Flat EEG to be studied.

\section{Materials and Methods}

This section will present the dynamic model construction for Flat EEG. Firstly, Flat EEG at any particular time is transformed into points. The collection of points are then viewed as a trajectory in a state space and hence modeled as a dynamical system.

\section{Geometrical Representation of Flat Electroencephalography}

A point is the fundamental element of dynamical systems. Thus, to model Flat EEG as dynamical system, a new representation of Flat EEG must first be built. Basically, a geometrical representation i.e., a point will be used to represent Flat EEG. In this new representation, Flat EEG at any time is transformed into a $n$-tuples where $n$ depends on the total number of information contained in Flat EEG.

Flat EEG at any time displays the average electrical potential readings from each of the sensors. This information must be taken into account when transforming to a point. That is, it must be retained in the new representation. Generally, the Flat EEG of an EEG 
signal recorded using $n$ sensors will be represented as an $n$-tuples in $n$-dimensional Euclidean space whereby $n$ accounts for all the sensors (Fig. 8). Consider the flattening mapping:

$$
S_{t}\left((x, y, z), e_{p}\right)=\left(\frac{r x}{r+z}, \frac{r y}{r+z}\right)_{e_{p}(x, y, z)}
$$

Then, at any time, Flat EEG can explicitly be denoted as:

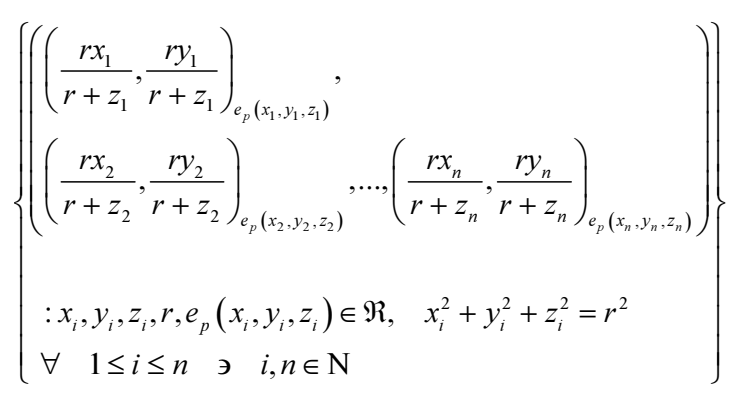

In this new representation, recorded electrical potential, denoted generally as $e_{p}\left(x_{n}, y_{n} z_{n}\right)$, is positioned on the right bottom of its corresponding sensor's coordinate location. Notice that the location of sensors placed on the head does not change throughout a recording of EEG signals. Thus, the complicated notation can certainly be reduced into a simpler form without losing the essential information. To do so, the coordinate location of sensors placed on the scalp will be removed and labeled on the axes of $\Re^{n}$ (Fig. 9 and 10). Meanwhile recorded electrical potential will take over its position. Hence, Flat EEG at any time can now be rewritten as:

$\left\{\begin{array}{l}\left(e_{p}\left(x_{1}, y_{1}, z_{1}\right), e_{p}\left(x_{2}, y_{2}, z_{2}\right), \ldots, e_{p}\left(x_{n}, y_{n}, z_{n}\right)\right) \\ : e_{p}\left(x_{i}, y_{i}, z_{i}\right) \in \mathfrak{R} \quad \forall \quad 1 \leq i \leq n \quad \ni \quad i, n \in \mathrm{N}\end{array}\right\}$

Therefore, a new geometrical representation of Flat EEG has been built. In this new form, Flat EEG at any time can be regarded as a point belonging to $\Re^{n}$, where $n$ depends on the number of EEG sensors (Fig. 11). Apart from being simple, it displays the numerical values of recorded electrical activity inside the brain visually. This new form which is the fundamental element of dynamical system will allow Flat EEG to be modeled as a continuous-time dynamical system.

\section{Model Construction}

It is reasonable and appropriate to model Flat EEG as a dynamical system because Flat EEG evolves over time. That is, real time is embedded within the data (Fig. 12).

To construct the model, it will firstly be assumed that Flat EEG is governed by a set of $n$ scalar differential equations with a unique solution whereby the solution for these set of differential equations will be the flow of the dynamical system of Flat EEG. Based on the definition of flow (Stephen, 2000), the mapping can then be written as.

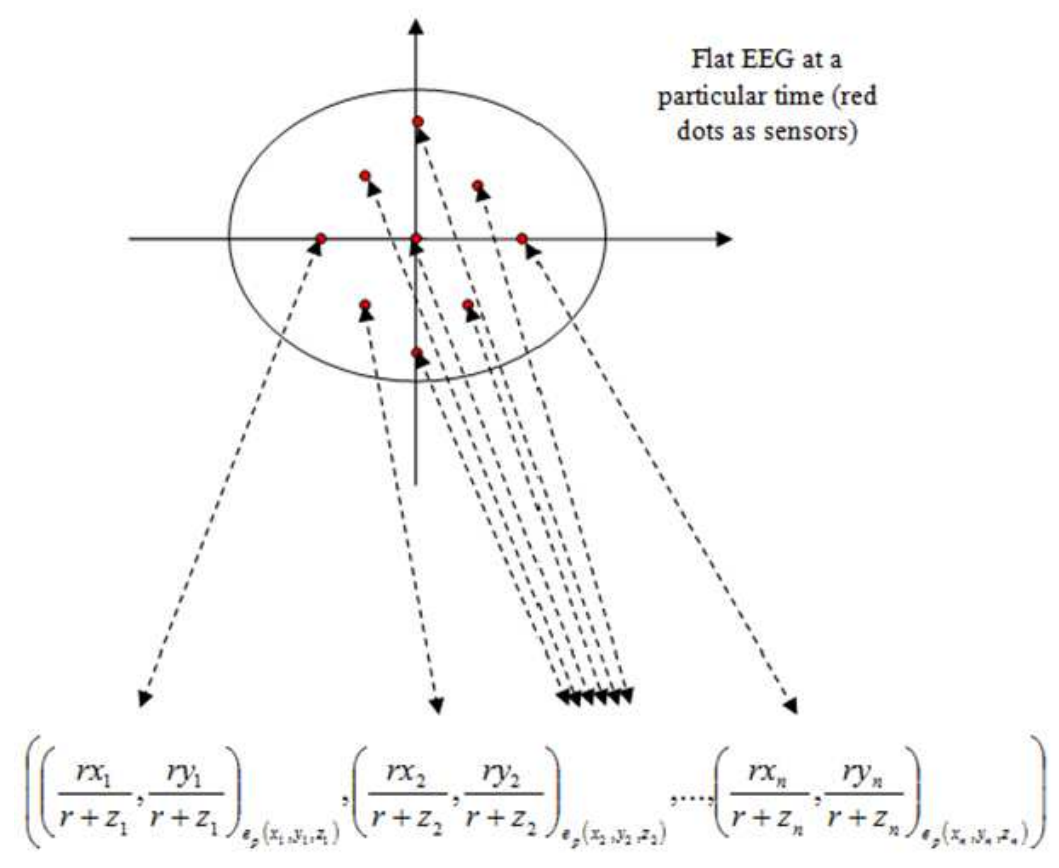

Fig. 8. Flat EEG at any time as $n$-tuples 


$$
\begin{aligned}
& \left\{\left(\left(\frac{r x_{1}}{r+z_{1}}, \frac{r y_{1}}{r+z_{1}}\right)_{e_{p}\left(x_{1}, y_{1}, z_{1}\right)},\left(\frac{r x_{2}}{r+z_{2}}, \frac{r y_{2}}{r+z_{2}}\right)_{e_{p}\left(x_{2}, y_{2}, z_{2}\right)}, \ldots,\left(\frac{r x_{n}}{r+z_{n}}, \frac{r y_{n}}{r+z_{n}}\right)_{e_{p}\left(x_{n}, y_{n}, z_{n}\right)}\right)\right\} \\
& \text { I } \\
& \left\{\begin{array}{l}
\left(e_{p}\left(x_{1}, y_{1}, z_{1}\right), e_{p}\left(x_{2}, y_{2}, z_{2}\right), \ldots, e_{p}\left(x_{n}, y_{n}, z_{n}\right)\right) \\
: e_{p}\left(x_{i}, y_{i}, z_{i}\right) \in \Re \quad \forall \quad 1 \leq i \leq n \quad \ni \quad i, n \in \mathrm{N}
\end{array}\right\}
\end{aligned}
$$

Fig. 9. Simplification of Flat EEG's $n$-tuples representation

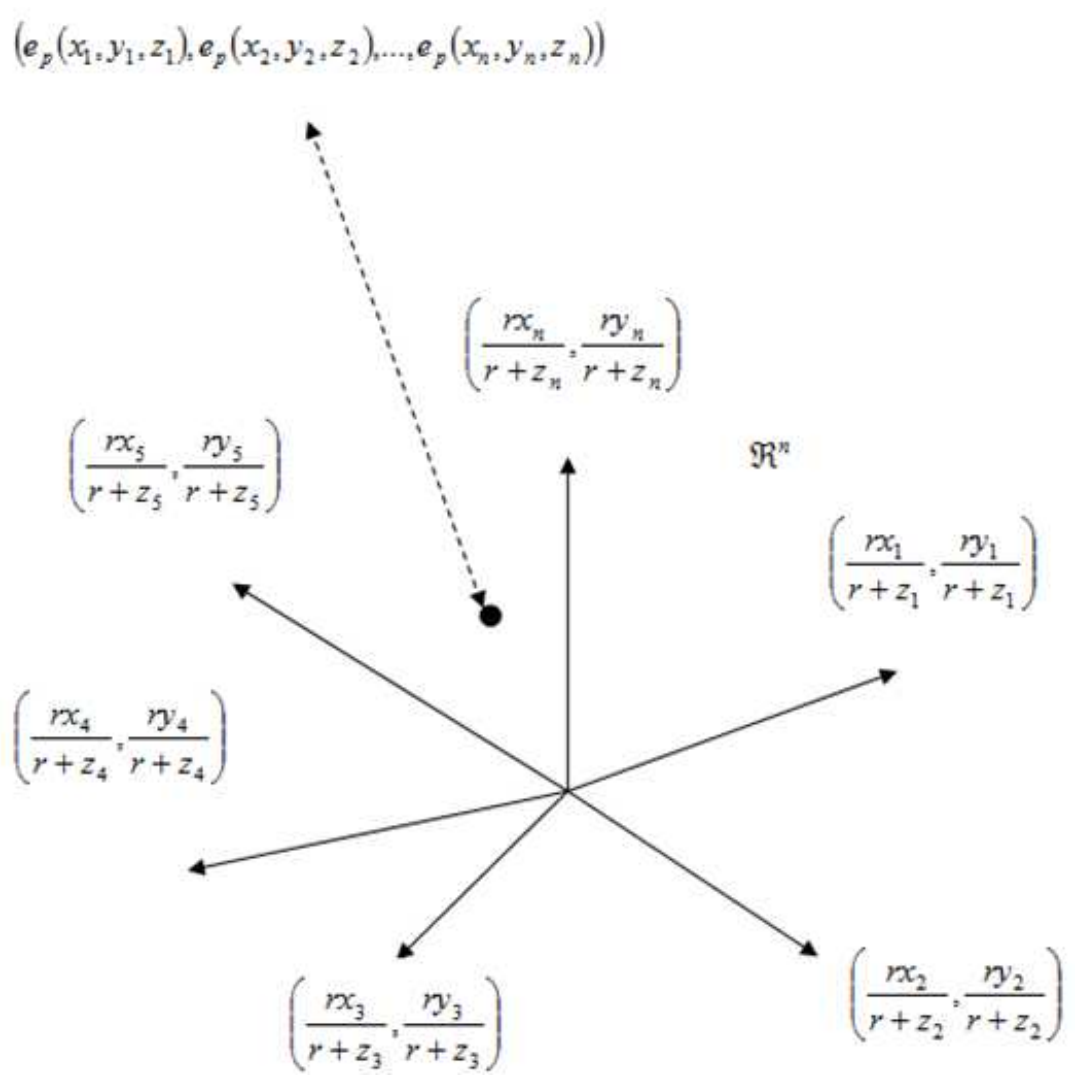

Fig. 10. Labeling of axes with sensors locations

$\psi_{t}(y)$ where, $\psi: \mathfrak{R} \times Y \rightarrow Y$ and such that the following two properties are fulfilled:

- $\psi_{0}(y)=y \forall y \in Y=\Re^{n}$ and

- $\quad$ For all $t$ and $s \in \mathfrak{R}$

- $\psi_{t}{ }^{\circ} \psi_{\mathrm{s}}=\psi_{t+s}$

For any $y_{k} \in Y=\mathfrak{R}^{n}, \psi_{t}\left(y_{k}\right)$ defines a curve in $Y$ when $t$ varies over $\mathfrak{R}$ (Fig. 13). This curve is also called as orbit or trajectory.
It represents a Flat EEG signal of an epilepsy patient who is having seizure. Distinct trajectories would correspond to different patients. Thus, the Flat EEG signal of any epilepsy patient who is having seizure can be represented as a trajectory in the state space (Fig. 14). Generally, a trajectory is the mapping:

$$
\psi_{t}: Y \rightarrow Y \text { where } \psi_{t}\left(y_{k}\right)=y_{t} \text { such that } \psi_{0}\left(y_{k}\right)=y_{k}
$$

where, by $\psi_{t}\left(y_{k}\right)=y_{t}$ is read as " $y_{t}$ is the state of the system at time $t$ initiated from $y_{k}$ ". 


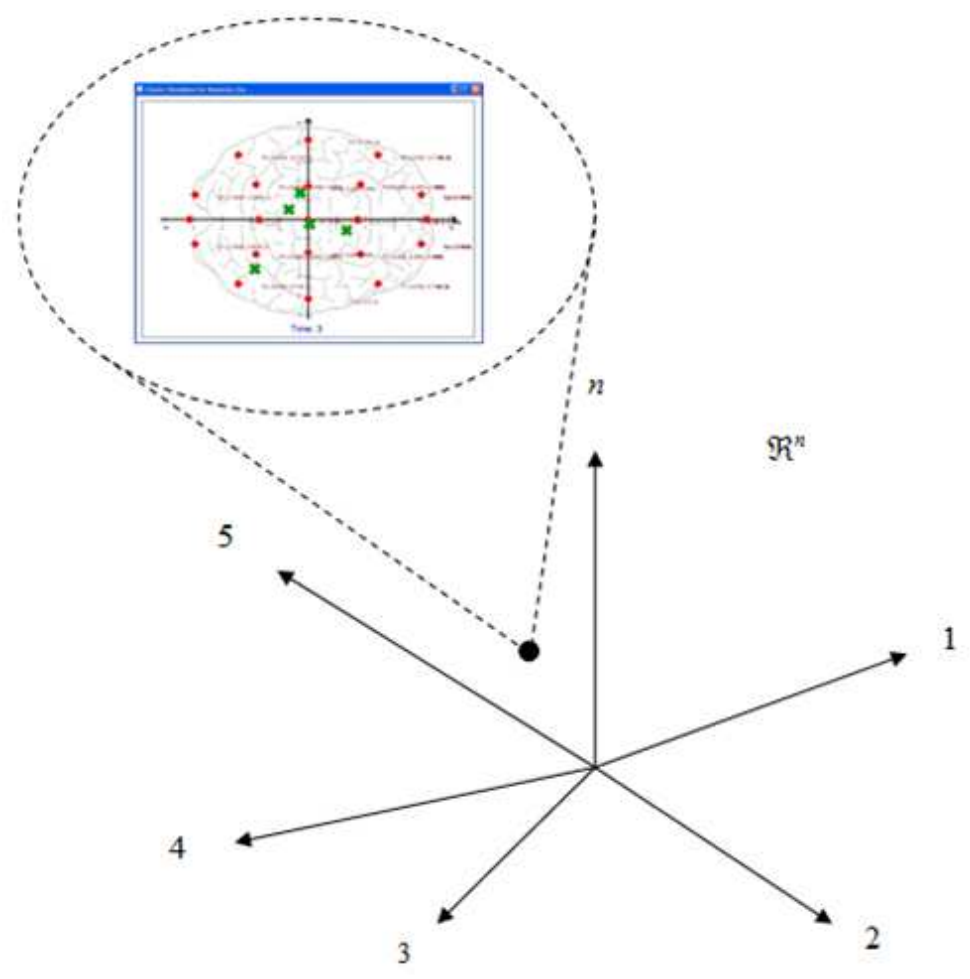

Fig. 11. Flat EEG as a point in $\mathfrak{R}^{n}$
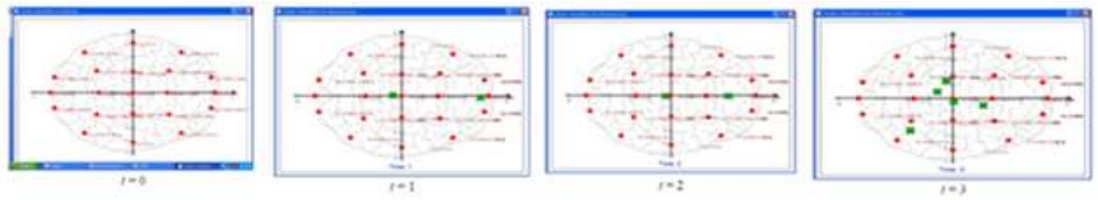

$\quad t=0$

$$
t=1
$$$$
t=2
$$$$
t=3
$$

Fig. 12. Embedment of real time in Flat EEG

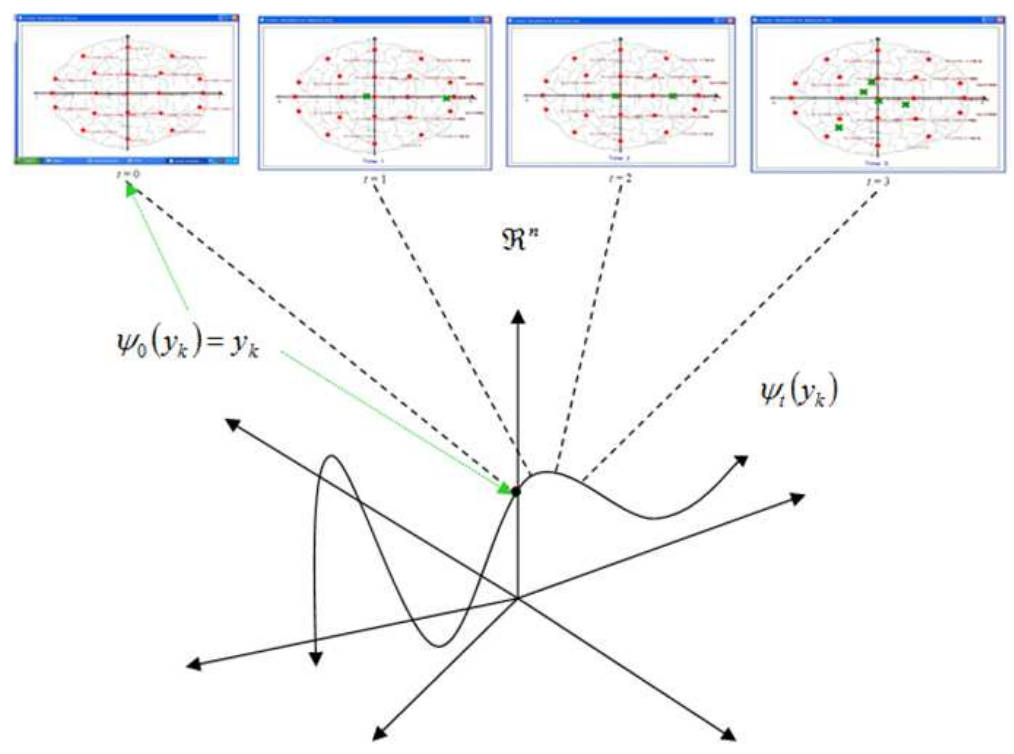

Fig. 13. Flat EEG as a point on trajectory 


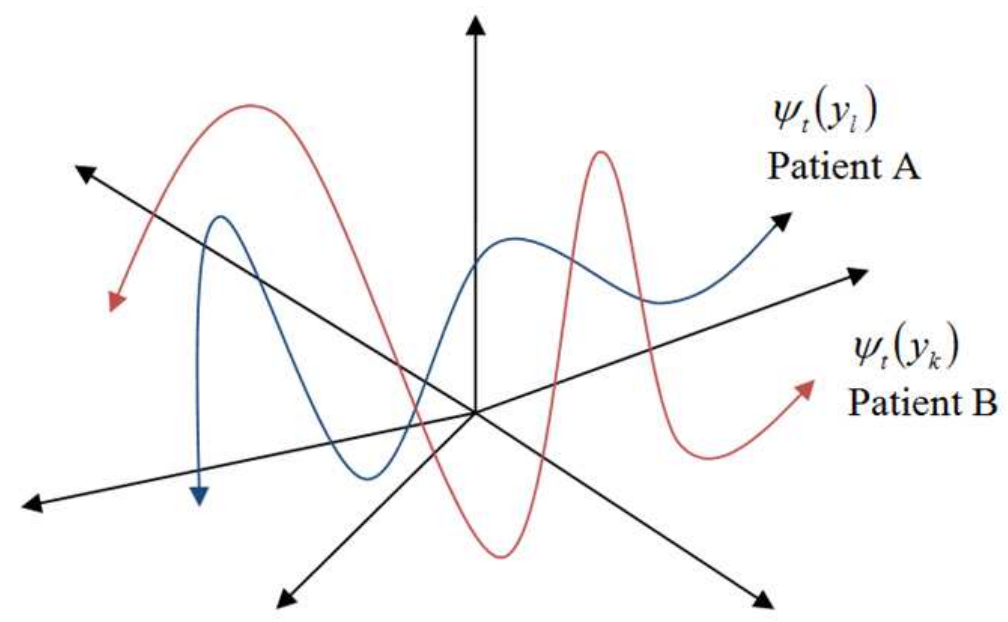

Fig. 14. Flat EEG of two different patients
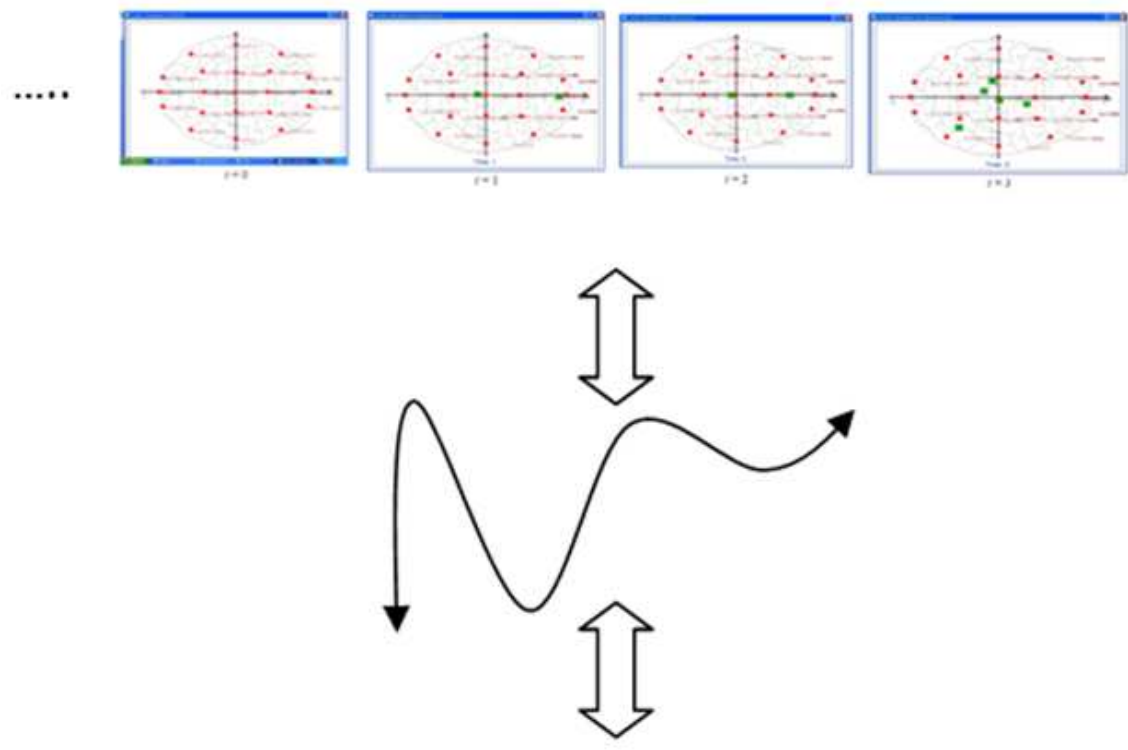

$O_{\psi_{t}\left(y_{k}\right)}$

Fig. 15. Event of Flat EEG in three different forms

Basically, the trajectory is a set of points and can be denoted as:

$O_{\psi_{t}\left(y_{k}\right)}=\left\{y_{t} \mid y_{t}=\psi_{t}\left(y_{k}\right) ; t \in \mathfrak{R}\right\}$

Recall that each Flat EEG at time $t$ can be written as a $n$-tuples (Equation 3). Thus, a trajectory (Equation 4) can be rewritten as:

$O_{\psi_{t}\left(y_{k}\right)}=\left\{\begin{array}{l}\left(e_{p}\left(x_{1}, y_{1}, z_{1}\right), e_{p}\left(x_{2}, y_{2}, z_{2}\right), \ldots, e_{p}\left(x_{n}, y_{n}, z_{n}\right)\right) \\ : e_{p}\left(x_{i}, y_{i}, z_{i}\right) \in \mathfrak{R} \quad \forall \quad 1 \leq i \leq n \quad \ni \quad i, n \in \mathrm{N}\end{array}\right\}$ where, $\left(e_{p}\left(x_{1}, y_{1}, z_{1}\right), e_{p}\left(x_{2}, y_{2}, z_{2}\right), \ldots, e_{p}\left(x_{n}, y_{n}, z_{n}\right)\right)$ represents Flat EEG at time, $t$ with $e_{p}\left(x_{i}, y_{i}, z_{i}\right)$ the electrical potential recorded from sensor $\left(x_{i}, y_{i}, z_{i}\right)$ (Fig. 15).

\section{Results}

Flat EEG at any time can be composed into points. As a whole, it can be viewed as a trajectory in a state space. Furthermore, the collections of all these trajectories can be regarded to be governed by a flow and hence be modeled as dynamical system. 


\section{Discussion}

Epileptic seizure is a very complex process due to its nature in exhibiting high variability i.e., high number of state variables (Sergio et al., 2006). However, Flat EEG which provides a reflection on the dynamics of electrical activity in populations of neurons can be a good platform to study epileptic seizure. In fact, there have been numerous researches utilizing various mathematical tools to visualize and extract "hidden" information within Flat EEG signals. While most of these have resulted in promising outcomes the indisputably essential theoretical justification for Flat EEG as an alternative platform in representing epileptic seizure dynamically has not been constructed. It is anticipated that the establishment of dynamic model on Flat EEG in this study would contribute to the construction of dynamic transformation from epileptic seizure to Flat EEG and hence justifying Flat EEG theoretically. Apart from that, the structural stability of Flat EEG could also be studied.

\section{Conclusion}

In this study, a geometrical representation i.e., a point, of Flat EEG at any time was formulated. This new representation displays the numerical values of recorded electrical activity inside the brain more visibly as compared to the figure form of Flat EEG (recall Fig. 3). Besides, the trajectory form of Flat EEG signals is also formed whereby the coordinates of each point on the trajectory displays the magnitude of electrical potential from each axes. Eventually, a dynamic model was established on Flat EEG for forthcoming analysis.

\section{Acknowledgement}

The authors would like to thank their family members and friends, especially Ong Sie Meng, for their continuous support and assistance. Authors also appreciate the financial support received from grant Vot. RDU 141307 and Potential Academic Staff grant (Q.K130000.2743.01K75) by UTM.

\section{Author's Contributions}

Tan Lit Ken: Designed the research plan and organized the study. Coordinated the overall framework and contributed to the writing of the manuscript.

Tahir bin Ahmad: Designed the research plan. Contributed in giving ideas and reviewing the manuscript critically.

Mohd Sham bin Mohd: Participated in reviewing the manuscript and giving ideas.

Su Kong Ngien: Participated in giving ideas, reviewing and proofreading the manuscript.

\section{Ethics}

This article is original and contains unpublished material. The corresponding authors confirms that all of the authors have read and approved the manuscript and no ethical issues involved.

\section{References}

Basar, E., E.C. Basar and J. Roschke, 1988. Do coherent patterns of the strange attractor EEG reflect deterministic sensory-cognitive states of the brain? Springer Series Synergetics, 39: 297-306.

Bennett, T.L., 1992. Ciritical issue in Neuropsychology: The Newropsychology of Epilepsy. 2nd Edn., Springer, pp: 88.

Burghes, D.N., P. Galbraith, N. Price and A. Sherlock, 1996. Mathematical Modelling. 1st Edn., Prentice Hall, pp: 1.

Desco, M., J. Pascau, M.A. Pozo, A. Santos and S. Reig et al., 2001. Multimodality localization of epileptic foci. Medical Imaging, 4321: 362-370.

Eduard, G., L. Helwig and W. Raine, 1999. Visualization of dynamical systems. Future Generat. Comput. Syst., 15: 75-86. DOI: $10.1016 / \mathrm{S} 0167-739 \mathrm{X}(98) 00054-5$

Edwards, D. and M. Hamsaon, 2001. Guide to Mathematical Modelling. 2nd Edn., Palgrave Macmillan, ISBN-10: 033379446X, pp: 336.

Eugene, M.I., 2007. Dynamical System in Neuroscience. 1 st Edn., MIT Press, Cambridge, ISBN-10: 0262090430, pp: 441.

Evim, A., A.B. Canan, B. Haluk and Y. Bulent, 2006. Computational analysis of epileptic focus localization. Proceedings of the 24th IASTED International Conference on Biomedical Engineering, (CBE' 06), pp: 317-322.

Faisal, A.M.B. and A. Tahir, 2010. EEG signals during epileptic seizure as a semigroup of upper triangular matrices. Am. J. Applied Sci., 7: 540-544. DOI: 10.3844/ajassp.2010.540.544

Fauziah, Z., 2008. Dynamic profiling of electroencephalography data during seizure using fuzzy information space. PhD Thesis, Universiti Teknologi Malaysia, Skudai.

Fell, J., J. Roschke, K. Mann and C. Scaffner, 1996. Discrimination of sleep stages: A comparison between spectral and nonlinear EEG measures. Electroencephalography Clin. Neurophysiol., 98: 401-410. DOI: 10.1016/0013-4694(96)95636-9

Fowkes, N.D. and J.J. Mahony, 1994. An Introduction to Mathematical Modelling, 1st Edn., Wiley, Chichester, ISBN-10: 0471934224, pp: 447.

Gibbons, M.N., 1995. A Concrete Approach to Mathematical Modelling. 1st Edn., John Wiley and Sons, New York, ISBN-10: 1118030648, pp: 620. 
Jagat, N.K., 1988. Mathematical Modelling. 2nd Edn., New Age International, New York, ISBN-10: 812240006X, pp: 259.

Jahnecke, C.A.N., L. Schwarz, M.A. Sovierzoski, D.F.M. Azevedo and F.I.M. Argoud, 2007. C++ Video-EEG processing system with sights to the epileptic seizure detection. Proceedings of the World Congress on Medical Physics and Biomedical Engineering. Aug. 27-Sept. 1, pp: 1052-1055. DOI: $10.1007 / 978-3-540-36841-0251$

Jan, C.D.M., B. Fetsje, G. Pawel, A.S. Cezary and I.B. Maria et al., 2004. A maximum-likelihood estimator for trial-to-trial variations in noisy MEG/EEG data sets. IEEE Trans. Biomedical Eng., 51: 2123-2128. DOI: 10.1109/TBME.2004.836515

Kalman, D., 1997. Elementary Mathematical Models: Order Aplenty and a Glimpse of Chaos. 1st Edn., Cambridge University Press, ISBN-10: 0883857073, pp: 345 .

Kiymik, M.K., I. Guler, A. Dizibuyuk and M. Akin, 2005. Comparison of STFT and wavelet transform methods in determining epileptic seizure activity in EEG signals for real-time application. Comput. Biol. Med., 35: 603-616. PMID: 15809098

Liau, L.Y., 2001. Homeomorfisma Antara $S^{2}$ Dan $E^{2}$ Melalui Struktur Permukaan Riemann Serta Deduksi Teknik Pembuktiannya Bagi Homeomorfisma Pemetaan Topologi Topografi Kabur (FTTM), MSc Thesis, Universiti Teknologi Malaysia, Skudai.

Natasa, M., D. Malek, Y. Ilker and J. Prasanna, 2003. 3D source localization of epileptic foci integrating EEG and MRI data. Brain Topography, 16: 111-119. DOI: 10.1023/B:BRAT.0000006335.75534.78

Panayiotopoulos, C.P., 2010. Atlas of Epilepsies, 1st Edn., Springer Science and Business Media, New York, ISBN-10: 1848821271, pp: 2000.

Samarskii, A.A. and A.P. Mikhailov, 2002. Principles of Mathematical Modelling: Ideas, Methods, Examples. 1st Edn., CRC Press, ISBN-10: 9780415272810, pp: 360.

Sergio, A., J. Volker and K. Holger, 2006. Extreme Events in Nature and Society. 1st Edn. Springer Science and Business Media, Berlin, ISBN-10: 354028611X, pp: 368.
Sharon, R., I.M. David and Y. Shlomo, 2004. Anticonvulsant efficiency, behavioral performance and cortisol levels: A comparison of carbamazepine (CBZ) and a fatty acid compound (SR-3). Psychoneuroendocrinology, 29: 113-124. PMID: 14604595

Song, I.H., D.S. Lee and S.I. Kim, 2004. Recurrence quantification analysis of sleep electoencephalogram in sleep apnea syndrome in humans. Neurosci. Lett., 366: 148-153. PMID: 15276236

Stefan, H. and T.J. Feuerstein, 2007. Novel anticonvulsant drugs. Pharmacol. Ther., 113: 165-183. PMID: 17030393

Stephen, W., 2000. Introduction to Applied Nonlinear Dynamical Systems and Chaos. 1st Edn., Springer Science and Business Media, ISBN-10: 0387001778, pp: 843.

Tahir, A. and L.K. Tan, 2010. Topological conjugacy between seizure and flat electroencephalography. Am. J. Applied Sci., 7: 1470-1476. DOI: 10.3844 /ajassp.2010.1470.1476

Tahir, A., S.A. Rashdi, L.Y. Liau Z. Fauziah and W.A.R. Wan Eny Zarina, 2005. Homeomorphisms of Fuzzy Topographic Topological Mapping (FTTM). Matematika, 21: 35-42.

Tan, L.K. and A. Tahir, 2014. Structural stability of flat electroencephalography. Life Sci. J., 11: 165-170.

Thomasson, N., T.K. Hoeppner, C.L. Webber, Jr and J.P. Zbilut, 2001. Recurrence quantification in epileptic EEGs. Phsy. Lett. A, 279: 94-101. DOI: 10.1016/S0375-9601(00)00815-X

Tiihonen, J., R. Hari, M. Kjola, U. Nousiainen and M. Vapalahti, 2004. Localization of epileptic foci using a large-area magnetometer and functional brain anatomy. Ann. Neurol., 27: 283-290. PMID: 2327737

Toni, A., N. Aopa, S.H. Matti, P.J. Liro and L. Jouko et al., 2005. Bayesian analysis of the neuromagnetic inverse problem with $1(\mathrm{p})$-norm priors. Neuroimage, 26: 870-884. PMID: 15955497

Wyllie, E., A. Gupta and D.K. Lachhwani, 2006. The treatment of epilepsy: Principles and Practice. 1st Edn., Lippincott Williams and Wilkins, Philadelphia, pp: 1247. 